\title{
Space charge calculations for sub-three-dimensional particle-in-cell code
}

\author{
Leonid G. Vorobiev and Richard C. York \\ National Superconducting Cyclotron Laboratory, Michigan State University, East Lansing, Michigan 48824
}

(Received 28 July 2000; published 15 November 2000)

\begin{abstract}
A novel approach for modeling high-current, charged particle beams in a three-dimensional manner is introduced. While the integration of beam motion equations is done as in completely $3 \mathrm{D}$ particle-in-cell codes, the space charge forces are found by an approximately self-consistent inclusion of the transverse and longitudinal fields. The algorithm is dramatically faster than fully $3 \mathrm{D}$ algorithms with computational times comparable to 2D field solvers. In addition, a sparser spatial grid and fewer required macroparticles provide significantly reduced memory demands. The proposed sub-3D technique has been verified with good agreement with other independent algorithms.
\end{abstract}

PACS numbers: 41.75. $-\mathrm{i}, 29.27 .-\mathrm{a}, 02.70 .-\mathrm{c}, 02.60 .-\mathrm{x}$

\section{INTRODUCTION}

The general 3D particle-in-cell (PIC) formulation determines the evolution of a high-current beam of $N_{p}$ particles distributed in phase space $\left(x, x^{\prime}, y, y^{\prime}, z, z^{\prime}\right)$ at an initial time $(t=0)$ to some later time $(t>0)$ by integration, in a self-consistent manner, of the $3 N_{p}$ motion equations in normalized form (parameters such as $q, p, \gamma$, and $c$ are included within the $\mathbf{F}$ notation),

$$
\mathbf{X}_{k}^{\prime \prime}=\mathbf{F}^{\mathrm{ext}}\left(\mathbf{X}_{k}\right)+\mathbf{F}^{\mathrm{sc}}\left(\mathbf{X}_{k}\right), \quad k=1, \ldots, N_{p},
$$

where $\mathbf{X}_{k}=\left(x_{k}, y_{k}, z_{k}\right)$ are spatial coordinates, $\mathbf{F}^{\text {ext }}=$ $\left(F_{x}^{\text {ext }}, F_{y}^{\text {ext }}, F_{z}^{\text {ext }}\right)$ are the external focusing forces of the focusing lattice, and $\mathbf{F}^{\mathrm{sc}}=\left(F_{x}^{\mathrm{sc}}, F_{y}^{\mathrm{sc}}, F_{z}^{\mathrm{sc}}\right)$ are space charge forces of the beam. The $F_{x, y, z}^{\mathrm{sc}}$ are computed by (i) introducing a spatial grid covering the beam domain, (ii) obtaining the space charge density on the grid, (iii) calculating the grid potential by a Poisson solver, (iv) and deriving the space charge forces $F_{x, y, z}^{\mathrm{sc}}$ by interpolation throughout the domain.

Since 2D or 3D PIC simulations repeat steps (ii) and (iii) many times, they are quite computationally intensive and time consuming. The demands of 3D PIC simulations are particularly high since they require the additional longitudinal dimension and a larger population of macroparticles for accurate calculation of the space charge density. As a consequence, a $2 \mathrm{D}$ simulation requiring hours can require days when extended to 3D.

While calculation times of 3D PIC codes can be reduced by, for example, the use of fast field solvers, sparser grids with further smoothing of grid functions, utilization of beam bunch distribution symmetries, or parallel processing, the computational times are still relatively long.

To achieve significant reduction in computational times, we present a "sub-3D" PIC that incorporates a sub-3D field solver [1]. This approach provides a significant reduction in computational time without a concomitant loss of accuracy.

\section{COMPUTATIONAL TOOLS FOR SPACE CHARGE CALCULATIONS}

We want to find the space charge potential $u(x, y, z)$ of a beam propagating within a $3 \mathrm{D}$ region $\Re$. Note that throughout this paper we consider only the purely electrostatic case neglecting Coulomb collisions.

The 3D Poisson equation may be written as

$$
\begin{aligned}
\Delta u & =-4 \pi \rho, \quad(x, y, z) \in \Re, \\
\left.u(x, y, z)\right|_{\partial \Re} & =U_{0}(x, y, z),
\end{aligned}
$$

where $\rho(x, y, z)$ is the $3 \mathrm{D}$ charge density and $U_{0}$ is the boundary potential. For example, in the particular case where $\mathfrak{R}$ represents a round metal cylinder with a boundary $\partial \Re, U_{0}=0$ omitting wakefield effects.

As a specific example, we consider a beam bunch with a total charge of $10^{-11} \mathrm{C}$ uniformly distributed inside a circular conducting chamber $4 \mathrm{~cm}$ in diameter, as shown in Fig. 1. The pearlike shape of the beam bunch was

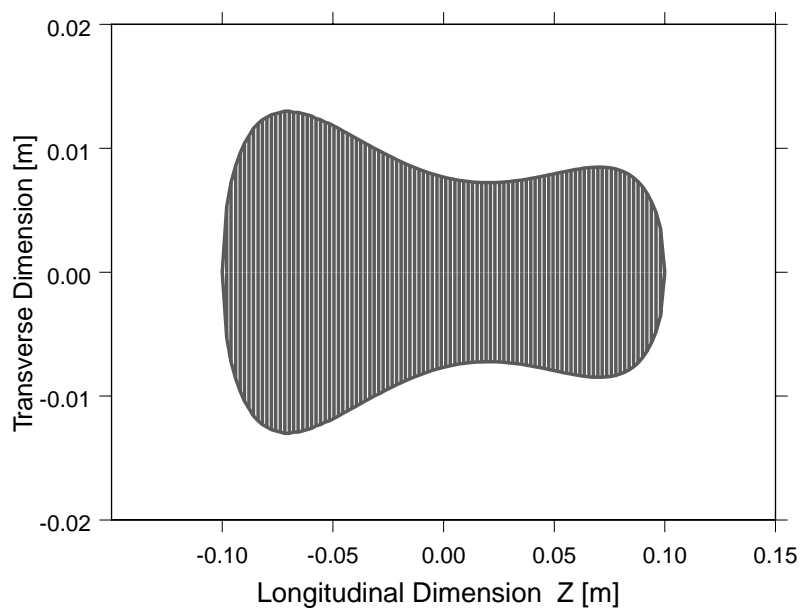

FIG. 1. Beam bunch with total charge of $10^{-11} \mathrm{C}$ and a longitudinally asymmetric charge density inside a round metal pipe $4 \mathrm{~cm}$ in diameter. 


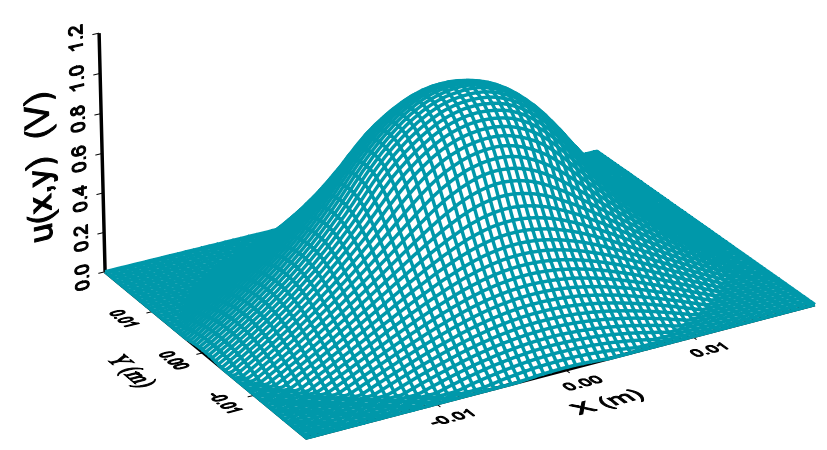

FIG. 2. (Color) The space charge potential $u(x, y, z=0)$ calculated by the SOR-3D method for the bunch of Fig. 1.

chosen especially to emphasize the nonlinear effects of the self-electric fields.

\section{A. General successive over-relaxation 3D (SOR-3D)}

A general successive over-relaxation, three-dimensional (SOR-3D) method was used as a point of comparison for the techniques presented in this paper. The space charge potential of the beam bunch of Fig. 1 was calculated using the SOR-3D algorithm. The horizontal $(x)$, vertical $(y)$, and longitudinal $(z)$ grid sizes were $N_{x}=90, N_{y}=90$, and $N_{z}=300$, respectively. In this example, the charge was distributed uniformly resulting in a constant grid density within the beam of Fig. 1. The calculated 3D potential in the middle cross section of the beam $(z=0)$ is given in Fig. 2. We show the 3D potential data as $u(r, z)$ for different radii in Fig. 3.

\section{B. Slice algorithm}

In previous papers [1-3], a numerical technique was introduced for calculating the longitudinal $(z)$ space charge potential by superposition of the potentials from thin disks (slices). This procedure was found to give an accurate
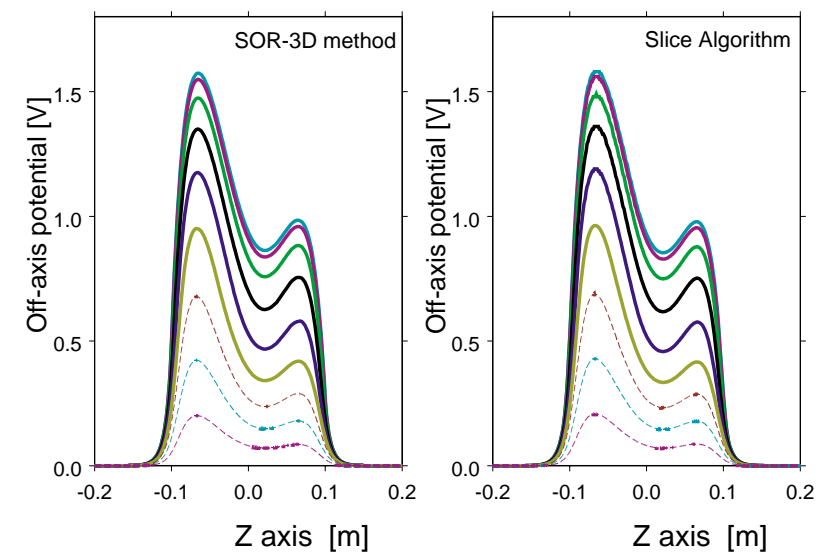

FIG. 3. (Color) The space charge potential $u(r, z)$ as a function of $z$ for different radii for the bunch of Fig. 1 inside a conducting pipe of radius $2 \mathrm{~cm}$ calculated using the SOR-3D method (left) and the slice algorithm (right).

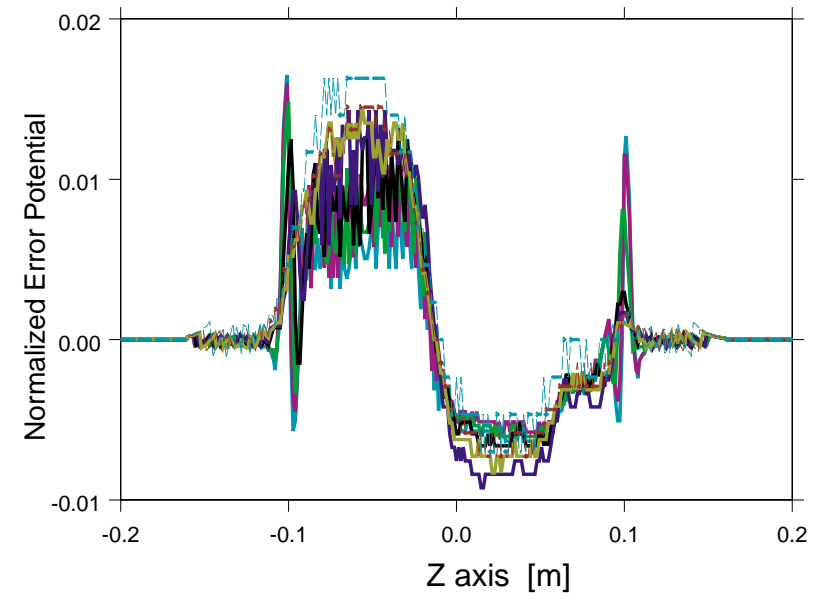

FIG. 4. (Color) Relative error $\left(u_{\text {slice }}(r, z)-u_{\text {SOR }}(r, z)\right) /$ $u_{\max }(r, z)$ between the slice algorithm and SOR-3D results of Fig. 3. The maximal error is within $1.6 \%$.

representation of the longitudinal fields while being computationally very fast.

For the configuration of Fig. 1, but using the slice technique with the beam bunch represented as a sequence of 100 thin disks, the longitudinal space charge potential was also calculated. Shown in the left-hand side of Fig. 3 is $u(r, z)$ found by the SOR-3D algorithm and on the right-hand side found by the slice algorithm for radial coordinate $(r)$ values varying from 0 to $2 \mathrm{~cm}$. The potentials within the beam of Fig. 1 are shown as solid lines and those outside with dashed lines.

Shown in Fig. 4 is the normalized difference between the SOR-3D method and the slice algorithm. The agreement between the two procedures is better than $1.6 \%$. The space charge potentials for the same beam were also found by the SOR-3D method and compared with the slice algorithm for beam chamber with radii of 3 and $4 \mathrm{~cm}$. The coincidence between the two approaches is within $1 \%-2 \%$ for all cases.

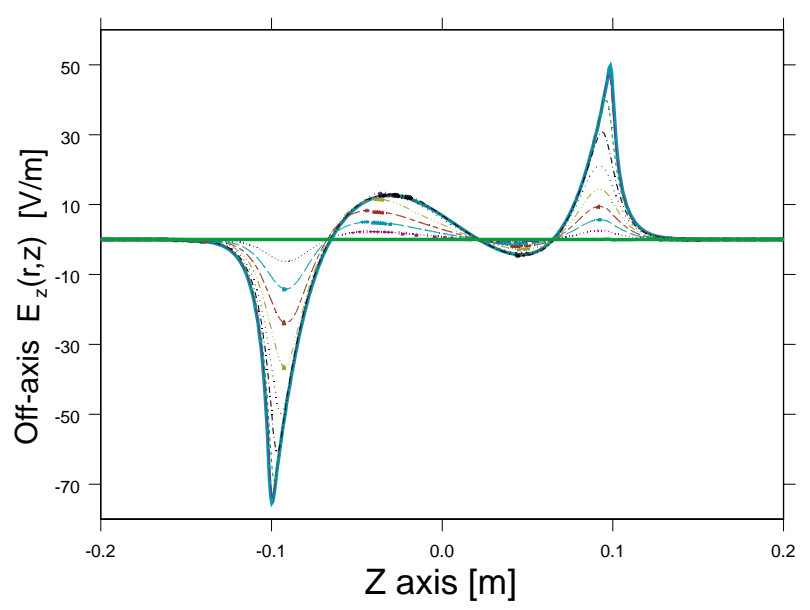

FIG. 5. (Color) Off-axis electric field $E_{z}(r, z)$ as a function of $z$ for different radii: $0 \leq r \leq 2 \mathrm{~cm}$ for the beam bunch of Fig. 1 . 


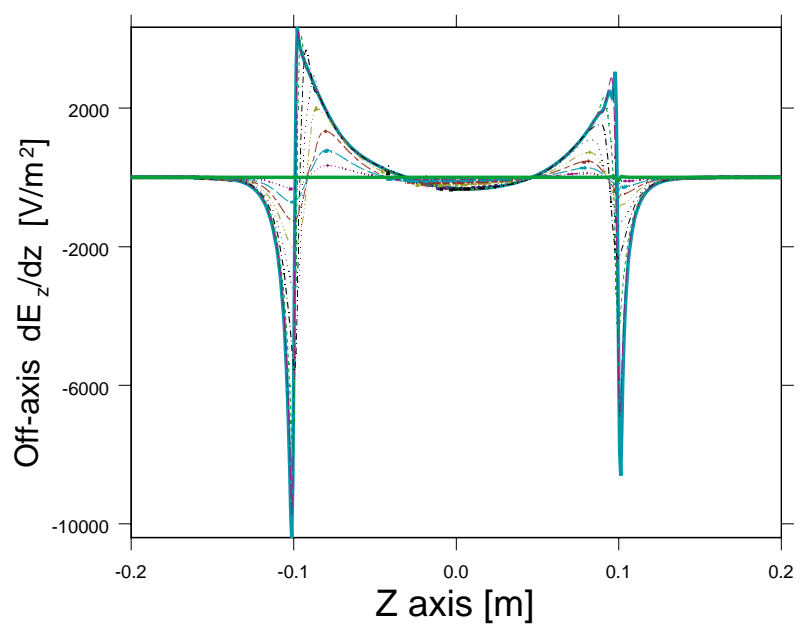

FIG. 6. (Color) Off-axis field derivatives $\partial E_{z} / \partial z$ as functions of $z$ for different radii: $0 \leq r \leq 2 \mathrm{~cm}$ for the beam bunch of Fig. 1.

The off-axis values of $E_{z}$ and $\partial E_{z} / \partial z$ as a function of " $z$ " are plotted in Figs. 5 and 6 for different " $r$." They were derived numerically from the potential data from Fig. 3 found by the slice algorithm.

The behavior of $E_{z}$ and $\partial E_{z} / \partial z$ is strongly nonlinear. In particular, the field $E_{z}$ changes sign four times, and, as a consequence, particles with different longitudinal coordinates would experience forces in opposite directions depending on their $z$ coordinate.

\section{SUB-3D POISSON SOLVER}

Writing the Laplace operator of Eq. (2) in Cartesian coordinates and moving the second derivative with respect to the longitudinal coordinate $(z)$ to the right-hand side, we have

$$
\frac{\partial^{2} u}{\partial x^{2}}+\frac{\partial^{2} u}{\partial y^{2}}=-4 \pi \rho-\frac{\partial^{2} u}{\partial z^{2}} .
$$

With the notation $\rho_{\text {corr }}(x, y, z)=\rho+\left(\partial^{2} u / \partial z^{2}\right) / 4 \pi$, one obtains

$$
\frac{\partial^{2} u}{\partial x^{2}}+\frac{\partial^{2} u}{\partial y^{2}}=-4 \pi \rho_{\text {corr }} .
$$

If the space charge density function $\rho_{\text {corr }}(x, y, z)$ is known throughout the $\Re$ region, the solution of the original $3 \mathrm{D}$ problem (2) may be found by solving (3) for different $z$. Given complete knowledge of the driving term $\partial^{2} u / \partial z^{2}$, the sequence of solutions of the $2 \mathrm{D}$ problem of equation (3) will provide the same result as that of the general 3D solution of Eq. (2).

From the longitudinal potential $u(x, y, z)$ computed by the slice algorithm of Sec. II, the longitudinal field $E_{z}=$ $-\partial u / \partial z$ and its derivative $\partial E_{z} / \partial_{z}$ may be calculated numerically, as shown in Figs. 5 and 6. Since $\partial^{2} u / \partial z^{2}=$ $-\partial E_{z} / \partial z$, the "corrected" density ( $\left.\rho_{\text {corr }}\right)$ in Eq. (3) may be obtained.
Then the 3D space charge potential and fields may be found by (i) dividing the bunch into $N_{z}^{T}$ longitudinal slices ${ }^{1}$ with each slice possibly having a different thickness along the longitudinal $(z)$ dimension, depending on the bunch profile (see Fig. 7). (ii) For each slice with coordinate $Z_{T}$ and the thickness $H_{Z}^{T}$, finding the 2D density $\rho_{2 \mathrm{D}}(x, y)$, taking into account only those particles associated with the specific slice. (iii) For each slice, finding the corresponding spatial 3D density

$$
\begin{aligned}
\rho_{\text {corr }} & =\rho(x, y, z)+(1 / 4 \pi)\left(\partial^{2} u / \partial z^{2}\right) \\
& =\rho_{2 \mathrm{D}}(x, y) / H_{Z}^{T}+(1 / 4 \pi)\left(\partial^{2} u / \partial z^{2}\right) .
\end{aligned}
$$

(iv) Solving the $2 \mathrm{D}$ Poisson equation (3) for each slice using the corrected density $\rho_{\text {corr }}$ found by evaluation of the driving term $\partial^{2} u / \partial z^{2}=-\partial E_{z} / \partial z$ calculated by the slice algorithm.

By modeling the 3D potential of the beam in this manner, it is possible to simulate the full 3D dynamics since the algorithm operates with the full range of charged particles in $\left(x, x^{\prime}, y, y^{\prime}, z, z^{\prime}\right)$ and the particles within the beam bunch can move in all three directions drifting from one "thick" slice to another.

The sub-3D Poisson solver is used to find the $N_{Z}^{T}$ solutions of the 2D Poisson solver with corrected space charge density, derived from the slice technique.

In brief,

$$
\begin{aligned}
F_{x, y, z}^{\mathrm{sc}}(x, y, z)[\mathrm{sub}-3 \mathrm{D}] & =F_{x, y}^{\mathrm{sc}}(x, y, z)[2 \mathrm{D}] \\
& \& F_{z}^{\mathrm{sc}}(x, y, z) \text { [slice algorithm]. }
\end{aligned}
$$

Although a computational economy may be achieved by appropriate variation of the slice thickness, in this paper we employ all slices of equal longitudinal $(z)$ thickness.

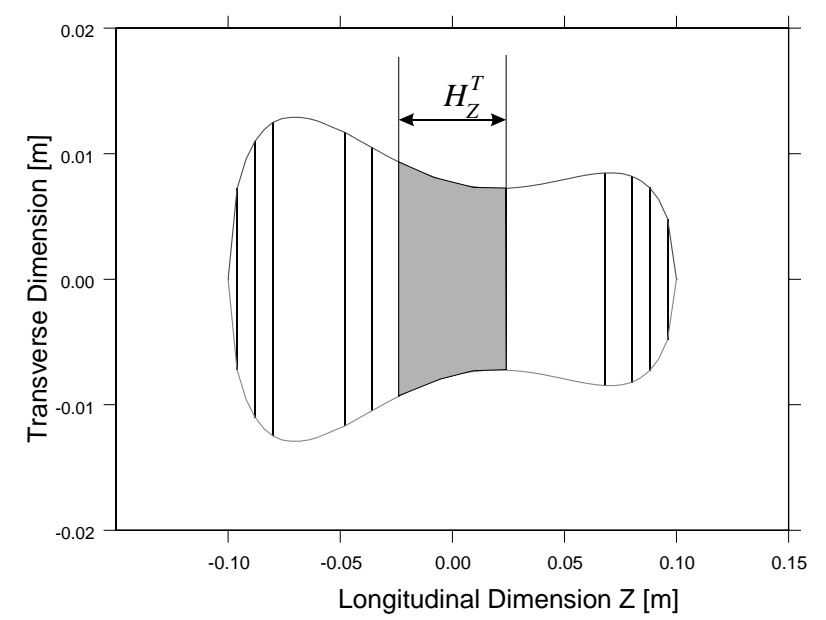

FIG. 7. Illustration of the sub-3D computational scheme. A specific thick slice with $z_{T}=0$ and width $H_{Z}^{T}$ is shaded.

\footnotetext{
${ }^{1}$ These thick slices should not be confused with the infinitesimally thin slices described in Sec. II for reconstructing the longitudinal beam potential since typically $N_{s}=50-100$, whereas $N_{Z}^{T}=5-10$.
} 

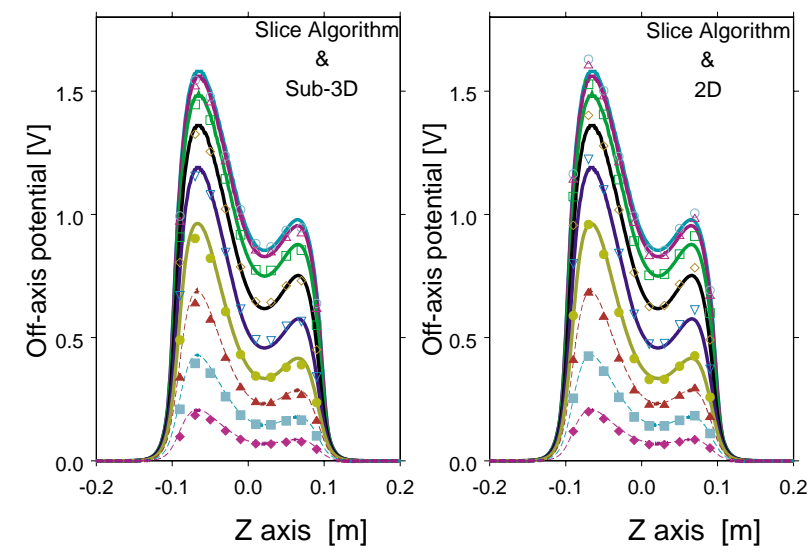

FIG. 8. (Color) On the left-hand side is the longitudinal potential of the beam bunch of Fig. 1 inside a metal pipe of radius $2 \mathrm{~cm}$ calculated by the slice algorithm of Sec. II (solid and dashed lines) and the sub-3D Poisson solver (symbols) for $0 \leq r \leq$ $2 \mathrm{~cm}$. On the right-hand side are the results of the sub-3D Poisson solver approach (symbols), but neglecting the $\partial^{2} u / \partial z^{2}$ correction term.

The potential of the beam bunch of Fig. 1 was evaluated using the sub-3D Poisson solver approach with $N_{Z}^{T}=10$ and $N_{p}=5 \times 10^{4}$.

Shown in Fig. 8 is a comparison between the potentials calculated by the slice algorithm of Sec. II (solid and dashed lines) and that obtained using the sub-3D Poisson solver approach (symbols). The number of thick slices is $N_{Z}^{T}=10$. The potentials within the beam are shown as solid lines (if within the bunch at least once) and those outside with dashed lines (if never within the bunch). On the right-hand side, one can see the results of the sub-3D Poisson solver approach (symbols) but neglecting the $\partial^{2} u / \partial z^{2}$ correction term. This mode of the sub-3D solver corresponds simply to the sequence of $2 \mathrm{D}$ solvers.
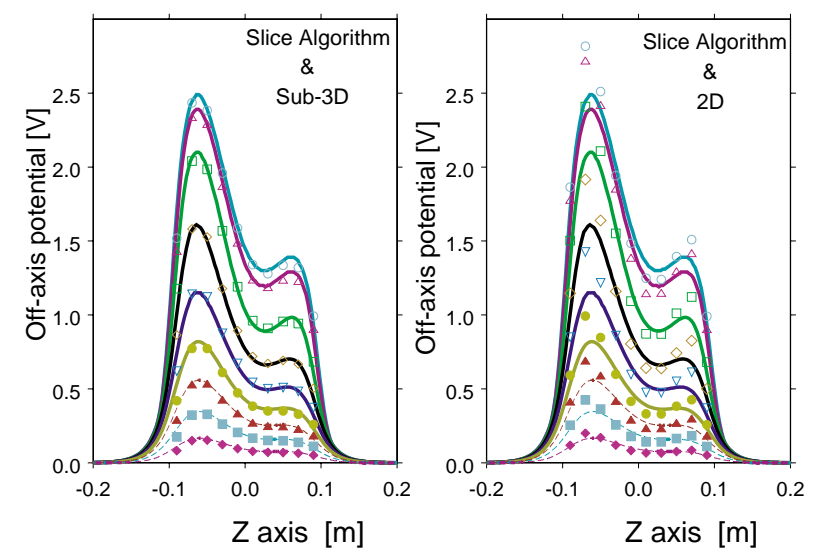

FIG. 9. (Color) On the left-hand side is the longitudinal potential of the beam bunch of Fig. 1 inside a metal pipe of radius $4 \mathrm{~cm}$ calculated by the slice algorithm of Sec. II (solid and dashed lines) and the sub-3D Poisson solver (symbols) for $0 \leq r \leq$ $4 \mathrm{~cm}$. On the right-hand side are the results of the sub-3D Poisson solver (symbols), but neglecting the $\partial^{2} u / \partial z^{2}$ correction term.

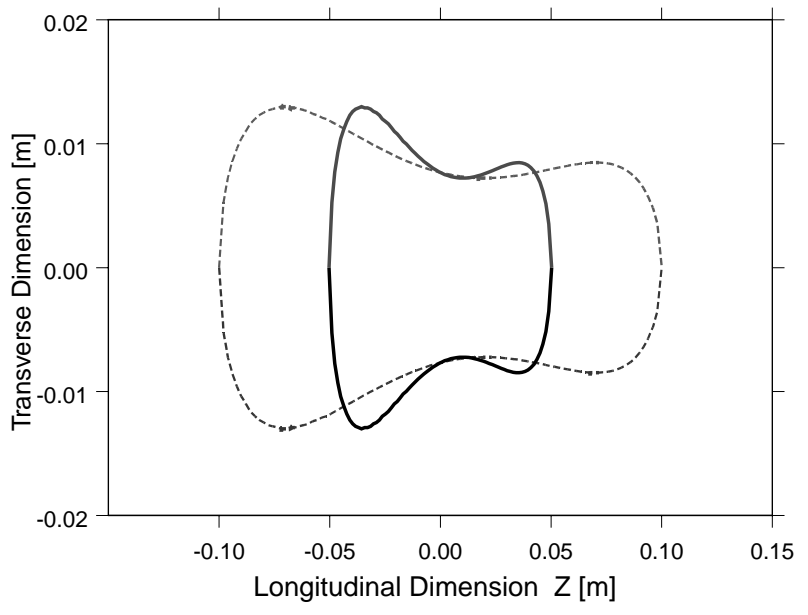

FIG. 10. The compressed beam bunch (solid line) inside a round metal pipe $4 \mathrm{~cm}$ in diameter, carrying the same space charge as the beam bunch of Fig. 1 (dashed line).

To evaluate the importance of incorporating the $\partial^{2} u / \partial z^{2}$ correction term, a similar analysis was also done with beam pipe radii of 3 and $4 \mathrm{~cm}$; see Fig. 9. For chamber radii of 2,3 , and $4 \mathrm{~cm}$, the maximum deviations between the sub-3D solver and the slice algorithm were $4 \%, 3 \%$, and 3\%, respectively, with the largest errors for regions outside of the bunch. However, omitting the $\partial^{2} u / \partial z^{2}$ driving term causes deviations of $4 \%, 15 \%$, and $21 \%$, respectively, with the largest errors within the bunch.

In a similar vein, a beam bunch with the same charge $\left(10^{-11} \mathrm{C}\right)$ and shape as that of Fig. 1, but longitudinally (z) shorter by a factor of 2, was evaluated; see Fig. 10. From Fig. 11, the sub-3D solver remains in good agreement when the driving term is included (left-hand plot), whereas the absence of the driving term (right-hand plot) shows a significant error of $20 \%$.
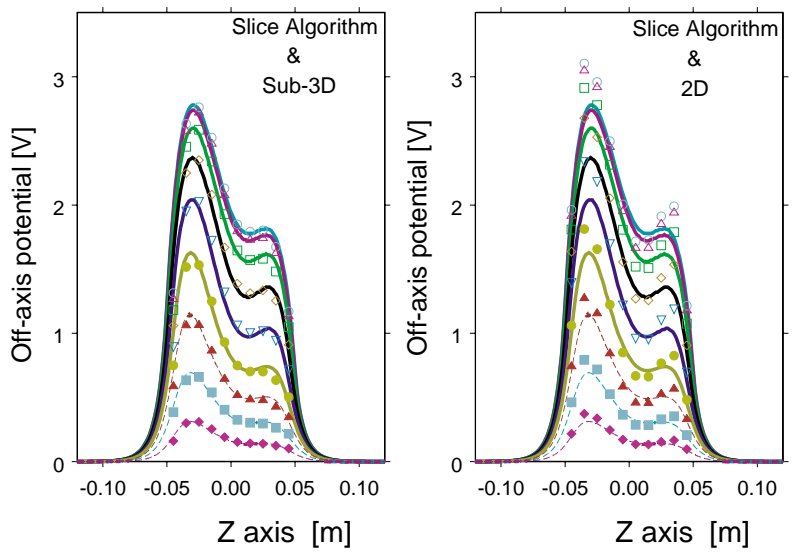

FIG. 11. (Color) On the left-hand side is the longitudinal potential of the beam bunch of Fig. 10 inside a metal pipe of radius $2 \mathrm{~cm}$ calculated by the slice algorithm of Sec. II (solid and dashed lines) and the sub-3D Poisson solver (symbols) for $0 \leq r \leq 2 \mathrm{~cm}$. On the right-hand side are the results of the sub-3D Poisson solver (symbols), but neglecting the $\partial^{2} u / \partial z^{2}$ correction term. 
For larger beam pipe diameter and shorter bunch lengths, the error caused by omission of the driving term was found to be even greater. For example, the case of a pipe radius of $4 \mathrm{~cm}$ (as in Fig. 9) and a compressed bunch (as in Fig. 10) resulted in an error of 30\%. Clearly then, care must be taken in the conditions under which the driving term is neglected.

\section{DISCUSSION AND CONCLUSION}

The numerical results obtained with the slice algorithm of Sec. II were compared to a general SOR-3D approach with agreement within a few percent. The numerical results of the slice technique were also compared to the sub-3D approach with agreement within several percent. The sub-3D approach in the absence of the driving term $\partial^{2} u / \partial z^{2}$ (corresponding to a series of $2 \mathrm{D}$ solutions) was found to produce significant errors in certain cases.

The goal of a relatively accurate, yet computationally efficient, algorithm has been met. The SOR-3D technique was used merely as a reliable tool for verification of the newer, faster algorithms. Though the SOR-3D procedure is very time consuming, and therefore unattractive for PIC applications, it is worth noting that, for examples given in this paper, computational times of $3-4 \mathrm{~h}$ on a $600 \mathrm{MHz}$ Alpha workstation were required. The examples in this paper using the sub-3D approach required only about $15 \mathrm{~s}$ on the same workstation if we solve Eq. (3) as a series of $2 \mathrm{D}$ problems assuming a round boundary, in which case SOR-2D is employed. If the round vacuum chamber is approximated by a rectangular pipe, the fast-Fouriertransform approach can be employed for the 2D part of the sub-3D solution with computational times of approximately $2 \mathrm{~s}$.

The increased computational speed of the sub-3D formulation is due to the reduced spatial grid dimensions and fewer particles. Namely, (i) instead of a longitudinal $(z)$ dimension of $N_{z}=128-256$ used in 3D models, the sub-3D approach utilizes $N_{Z}^{T}=5-10$ thick slices. The region over which the Poisson equation must be solved is limited in the $z$ direction by the beam bunch size. (ii) One to two orders of magnitude fewer particles are necessary for the sub-3D approach in comparison with 3D models since there is no need to reconstruct smooth longitudinal potentials and fields. The longitudinal field and its derivative, used by the sub-3D PIC, are supplied by the slice algorithm rather than calculated from the grid functions. Also, the reduced number of particles decreases the number of motion equations (1), significantly reducing the computation time for each integration step.

The sub-3D code takes the middle ground between 2D and 3D formulations. As with any 3D PIC code, the sub-3D PIC method operates with particles populating the six-dimensional phase space with the integration of the motion equations obtained in the same manner as for a completely $3 \mathrm{D}$ code. On the one hand, we borrow the $E_{z}(r, z)$ and $\partial E_{z} / d z$ values which are smooth enough due to the nature of the slice algorithm. On the other hand, the self-consistency of the whole model becomes approximate (for details see the theory of the slice algorithm in Refs. [2,3]), and this is the price for the high speed of calculations.

Though examples of this paper were limited to radially symmetric and constant charge density beam bunches propagating along the center of a conducting chamber, the principal idea of the sub-3D formulation may be applied more generally. In the near term, the code will be generalized to include beam bunches with arbitrary aspect ratios and charge density distributions.

\section{ACKNOWLEDGMENT}

This work was supported by the U.S. Department of Energy under Contract No. DE-FG02-99ER41118.

[1] L. G. Vorobiev and R. C. York, Michigan State University Report No. MSUCL-1149, 2000.

[2] L. G. Vorobiev and R. C. York, in Proceedings of the 1999 Particle Accelerator Conference, New York, edited by A. Luccio and W. MacKay (IEEE, Piscataway, NJ, 1999), p. 2781.

[3] L. G. Vorobiev and R. C. York, Michigan State University Report No. MSUCL-1117, 1998. 